\title{
Modified Glauber Model and a new interpretation of collective effects in AA and pA at LHC.
}

\author{
A.Seryakov ${ }^{* \dagger}$ \\ Saint Petersburg State University, Russia \\ E-mail: seryakov@yahoo.com \\ G.Feofilov \\ Saint Petersburg State University, Russia \\ E-mail: grigory-feofiloveyandex.ru
}

\begin{abstract}
The standard Glauber model is widely used for determination of centrality classes, for comparison of nucleus-nucleus and proton-nucleus collision with pp data and for search of collective effects. In the Modified Glauber Model (MGM) we take into account energy losses which are relevant to particle production in each nucleon-nucleon collision. MGM provides correct description of total multiplicity yields and scaling with number of nucleon-participants in nucleus-nucleus collisions from AGS to RHIC energies. MGM predictions of centrality dependence of multiplicity yields in $\mathrm{Pb}-\mathrm{Pb}$ collisions at LHC were confirmed by ALICE. MGM approach was transferred to $\mathrm{p}-\mathrm{Pb}$ collision and considerable stopping of nucleons was observed. Strong transformation of the behavior of multiplicity density and deviation of the scaling of soft and hard processes with number of nucleon-participants were obtained.
\end{abstract}

XXII International Baldin Seminar on High Energy Physics Problems,

15-20 September 2014

JINR, Dubna, Russia

\footnotetext{
* Speaker.

${ }^{\dagger}$ The work was supported by the St.Petersburg State University grant 11.38.193.2014.
} 


\section{Introduction}

The general approach in investigation of collective effects in nucleus-nucleus collisions is usually related to the problem of separation these effects from trivial background of independent nucleon-nucleon collisions. Two Component Model [1] and the Glauber approach [2] are widely used at present to estimate the number of inelastic nucleon-nucleon collisions and centrality in various experimental and theoretical works. The assumption was introduced in[1] that the multiplicity of hard and soft processes is proportional to the number of nucleon-nucleon collisions $\left(N_{\text {coll }}\right)$ and nucleon-participants $\left(N_{\text {part }}\right)$ correspondingly. In such a way that

$$
d N / d y=f n_{p p} N_{\text {part }}+(1-f) n_{p p} N_{\text {coll }}
$$

here $n_{p p}$ is the multiplicity measured in pp collisions per unit of (pseudo)rapidity at the given collision energy and $f$ is an adjustable parameter which could be different for different colliding systems and energies. The following fit to the data on the pseudo-rapidity density of charged multiplicity in non-single diffractive $p p$ and $p \bar{p}$ interactions at the given $\sqrt{s}$ is usually applied [3]:

$$
n_{p p}(s)=2.5-0.25 \ln (s)+0.023 \ln ^{2}(s)
$$

It was also assumed [1] that fitting the rapidity distribution in a nucleus-nucleus collisions by using Eq.1.1, 1.2 and the Glauber model one may obtain the correspondence between centrality and the mean number of participants. It was shown that the mean number of participants $N_{\text {part }}(n)$ in a nucleus-nucleus collision with multiplicity $n$ could be defined as [1]:

$$
N_{\text {part }}(n)=\frac{\int d^{2} b P(n ; b)\left(1-P_{0}(b)\right) N_{\text {part }}(b)}{\int d^{2} b P(n ; b)\left(1-P_{0}(b)\right)}
$$

here $n(b)$ - is the mean multiplicity of particles produced at a given impact parameter $\mathrm{b}$, evaluated analogously to Eq.1.1; $N_{\text {part }}$ (b) is the mean number of participants in the Glauber approach; and $P(n ; b)$ is the correlation function for $n(b)$ (see [1] for details).

$P_{0}(b)$ is the probability of no interaction among the nuclei at a given impact parameter b:

$$
P_{0}(b)=\left(1-\sigma_{N N} T_{A A}(b)\right)^{A A}
$$

where $\sigma_{N N}$ is the inelastic nucleon-nucleon cross section, and $T_{A A}(b)$ is the nuclear overlap function for the collision of two nuclei with atomic number A. Analogous to Eq.1.3 the formula for the mean number of nucleon-nucleon collisions $N_{\text {coll }}(n)$ can be obtained [1].

However, it is important to note that Eq.1.3 is writen with the assumption, that every nucleonnucleon collision takes place independently with the same energy $\sqrt{s}$ and cross-section $\sigma_{N N}$. One may expect that this last condition is an oversimplified. In particular, both the energy and the value of $\sigma_{N N}$ for subsequent inelastic elementary nucleon-nucleon collisions could change due to various effects. In particular, the particle production and the relevant energy loss by nucleon in the dominatingly "soft" nucleon-nucleon collision should lead to decrease of the value of nucleon momentum, to the following decreased value of $\sigma_{N N}$ for the next collision, and, therefore, finally, to change of values of $N_{\text {part }}$ and $N_{\text {coll }}$, and therefore Eq.1.3 becomes uncorrect. Thus one can expect that the resulting number of nucleon-nucleon collisions $N_{\text {coll }}$ could be overestimated in any 
standard approach that neglects these energy losses. In the present work we continue to investigate the last effect by the efficient account of the energy-momentum conservation included in updated version of the Monte Carlo algorithm of the Modified Glauber Model [4, 5]. The Modified Glauber Model (MGM) is introduced briefly in the 2st section and applied to the analysis of mean yields of charged multiplicities in a wide range of energies and centralities of nucleus-nucleus collisions. The 3nd and the 4th sections are devoted to comparison of MGM with SGM results for nucleusnucleus and proton-nucleus collisions in normalization of particles yields at the LHC respectively.

\section{Charged particles yield}

Connection between multiplicity of charge particles and the number of collisions in Standard Glauber Model is one of the approaches used in centrality estimates in heavy ion collisions. The naive solution is to start with mean charged particles multiplicity from the experimental pp data at a given collision energy and to fold with number of $N_{\text {coll }}$. In this picture the average multiplicity in a nucleus-nucleus collision is:

$$
<N_{c h}^{A A}>=<N_{\text {coll }}><N_{c h}^{p p}>
$$

where $N_{\text {coll }}$ is taken from the Glauber Model.

Naturally this naive method of superposition of nucleon-nucleon collisions does not work (see blue line for SGM results Fig. 1a). The widely used approach to eliminate the problem of exessive multiplicity in the Glauber model is to apply a so-called Two Components Model [1] in combination with the SGM. The majour drawback was mentioned in the introduction: it is the assumption of the fixed value of inelastic NN collisions cross-section $\sigma_{N N}$ for all nucleon-nucleon collisions. The alternative approach is based on the main idea that in each inelastic nucleon-nucleon collision some kinetic energy is lost for formation of particles. Therefore every next collision takes place in the simplified approach with lower energy and, correspondingly, with lower cross-section and multiplicity. For realization of this assumption the Modified Glauber Model was proposed in $[4,5]$ and updated for a study in the present work. Thus we take into account in an efficient way the collective effects in multiparticle production in nucleus-nucleus and proton-nucleus collisions just by consideration of energy needed to produce particles in all nucleon-nucleon collisions.

\section{The Modified Glauber Model}

In the Modified Glauber Model $[4,5]$ nucleons lose a fixed part $(1-k)$ of momentum in the center of mass system in each nucleon-nucleon collision.

$$
p^{\prime}=k p
$$

here $p$ - is a nucleon momentum in the center of two nucleons mass system before collision, $p^{\prime}$ a nucleon momentum after collision, $k$ - is the momentum loss parameter. Correspondingly every next collision of a given nucleon (or nucleon-like structure) with the new nucleons of incoming nucleus goes with the lower cross-section, and lower multiplicity of charge particles, which are taken from experimental data [7, 12]. In out Monte Carlo realization, in accordance with this 


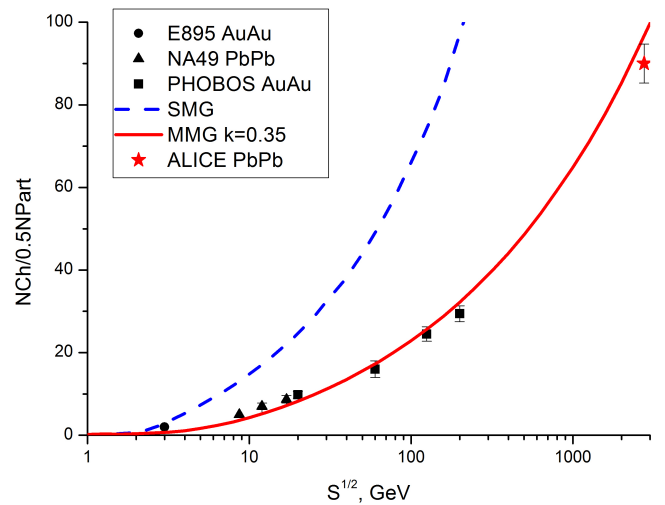

a)

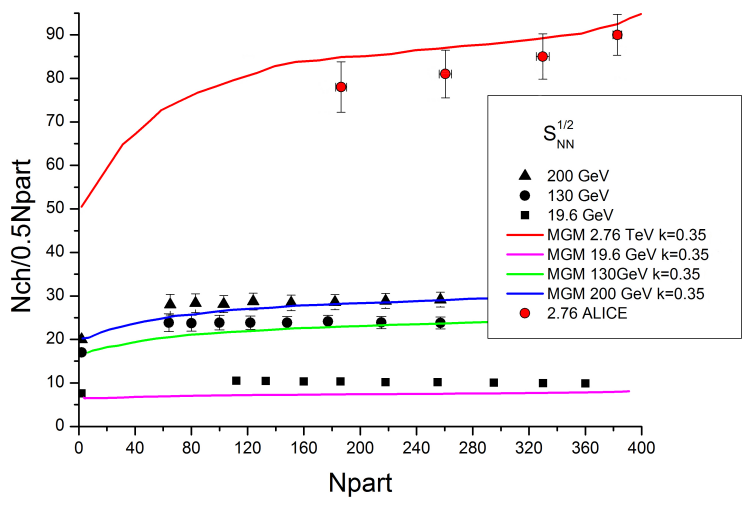

b)

Figure 1: a) Multiplicity for charged particles per the pair of nucleon-participants vs. the system of mass energy for the most central nucleus-nucleus collision. Black dots are experimental data (see compilation of data in [8]). The blue dashed curve is from SGM (see text). The red curve is the result of fitting the experimental data on nucleus-nucleus collisions for the region of collision energies from $19 \mathrm{GeV}$ to 200 GeV by MGM with $k=0.35$ and extrapolated to energy of $\sqrt{s}=2.76 \mathrm{TeV}$ of $\mathrm{Pb}-\mathrm{Pb}$ collisions at the LHC. The red star is the ALICE data from $\mathrm{Pb}-\mathrm{Pb}$ collisions at $2.76 \mathrm{TeV}$ [10]. $b$ ) Multiplicity for charged particles to the pair of nucleon-participants versus centrality of nucleus-nucleus collisions, defined by $N_{\text {part }}$. The red points are ALICE data [9] at 2.76 TeV. The black points are RHIC and NA49 data at three collision energies [8]. The curves are calculated in MGM with $k=0.35$ at relevant collision energies (no fits).

approach, the possibility of collisions between nulceons of the same nucleus was introduced. This can happen when a nucleon may lose all its momentum after several collisions and even may change the direction of its flight to opposite. The effect is negligible for AA but plays a big role for peripheral proton-nucleus collisions.

Parameter $k$ in our calculations was obtained by fitting the experimental multiplicity data in the most central $\mathrm{Pb}-\mathrm{Pb}$ and $\mathrm{AuAu}$ collision in a wide range of energy from 17 to $200 \mathrm{GeV}$ (see red curve in Fig. 1a).

\section{Nucleus-nucleus collisions}

The MGM with a single (fixed in the analysis) parameter $k$, which was fitted only for central events, describes multiplicity data for all centrality intervals in a broad range of $\mathrm{Au}-\mathrm{Au}$ and $\mathrm{Pb}-\mathrm{Pb}$ collision energies (from $19 \mathrm{GeV}$ to $2.76 \mathrm{TeV}$ ) including the new LHC data (Fig. 1b).

One may see that in the case of nucleus-nucleus collisions at the LHC the MGM predicts practically the same value of nucleon-participants $N_{\text {part }}$ as the SGM. But it is obtained for central $\mathrm{Pb}-\mathrm{Pb}$ collisions that the value of $N_{\text {coll }}$ is almost three times lower then in SGM due to the account of nucleon momentum loss done in the MGM (Fig. 2). This means that caution should be taken in any normalization of yields in the domain of soft processes of multiparticle production on results from standard Glauber model.

\section{Nucleon stopping in proton-nucleus collisions}

Proton-nucleus (pA) collisions are characterized by a strong asymmetry of colliding systems. 

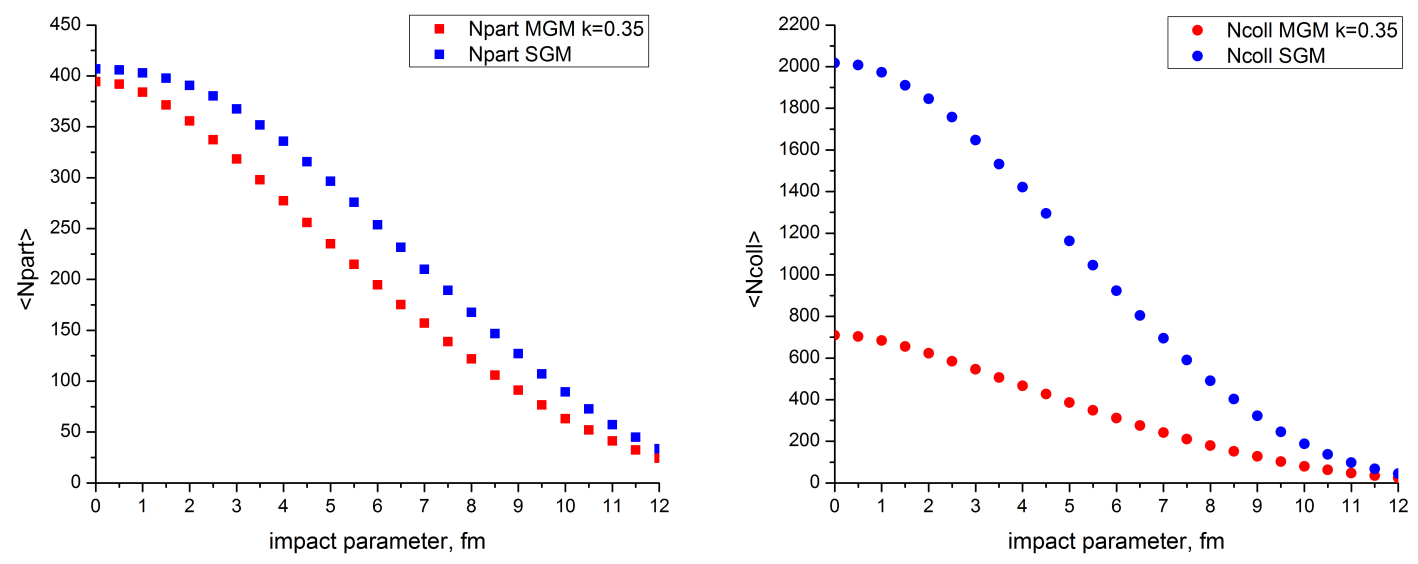

Figure 2: The average $N_{\text {part }}$ and $N_{\text {coll }}$ versus impact parameter for $\mathrm{Pb}-\mathrm{Pb}$ collisions at $\sqrt{s}=2.76 \mathrm{TeV}$ from the Standard Glauber Model (blue dots) and the Modified Glauber Model with $k=0.35$ (red dots).

In this case we have a simple relation between the number of participants and binary collisions:

$$
N_{\text {part }}=N_{\text {coll }}+1
$$

In the Figure 3(a) the MGM results with $k=0.35$ on the average numbers of $N_{\text {part }}$ and $N_{\text {coll }}$ in $\mathrm{p}-\mathrm{Pb}$ collisions at $\sqrt{s}=5.04 \mathrm{TeV}$ are presented versus impact parameter " $\mathrm{b}$ " and compared to the SGM calculations. First of all, dramatic difference with the standard Glauber (SGM) calculations is observed. One may see, that in this simplified picture, within the limits of MGM, a projectile proton does not have enough energy to pass through lead nucleus due to energy losses for charged particles production leading to nucleon stopping effect. Therefore about three times decrease of $N_{\text {part }}$ and $N_{\text {coll }}$ for zero impact parameter in comparison to SGM is observed. We may also note that in peripheral region the mean number of participants in MGM is larger than in SGM due to additional collision between nucleons considered in the updated model (some nucleons may stop the flight after considerable momentum loss and may be collided by overtaking nucleons from the same nucleus).

In view of these results, the normalization of proton-nucleus collisions data on the pseudorapidity density of charge particles in p-Pb collision performed in [6] should be reconsidered. The experimental values of pseudorapidity density of charge particles in proton(deutron)-nucleus collisions, normalized to the average $N_{\text {part }}$, are shown vs. collision energy per nucleon pair in Fig.3(b) together with some approximations. Compilation of all experimental data, except green points for $\mathrm{p}-\mathrm{Pb}, \mathrm{pAu}$ and $\mathrm{d}-\mathrm{Au}$ collisions, is taken from [6]. Red points are obtained in the experimental paper by using SGM for normalization of data in $\mathrm{p}-\mathrm{Pb}, \mathrm{p}-\mathrm{Au}$ and $\mathrm{d}-\mathrm{Au}$ collisions. Green points are recalculated values obtained in the present study from MGM with $k=0.35$.

Similar significant change of understanding the experimental data could be referred to the ratio of Z-bosons yields and charged-particle multiplicity [11] (see Fig. 4). These data mean that the given ratio is independent of the number of nucleon-participants, pointing at scaling with $N_{\text {part }}$. This result is based on the standard Glauber model estimate of $N_{\text {part }}$ in p-Pb collisions at LHC. 


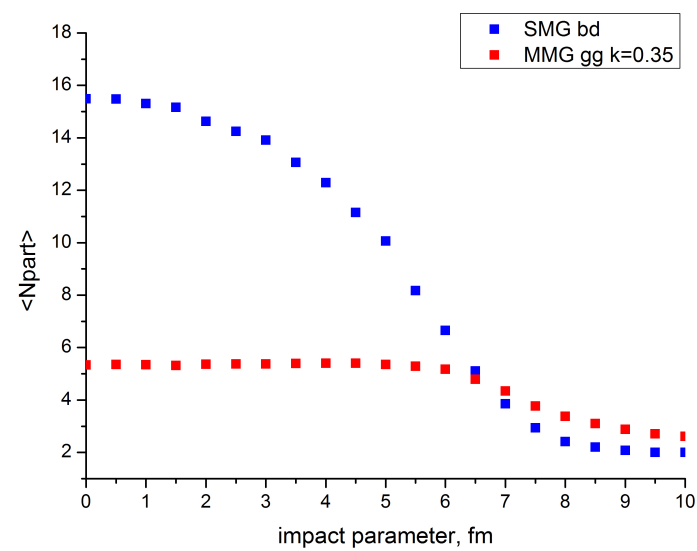

a)

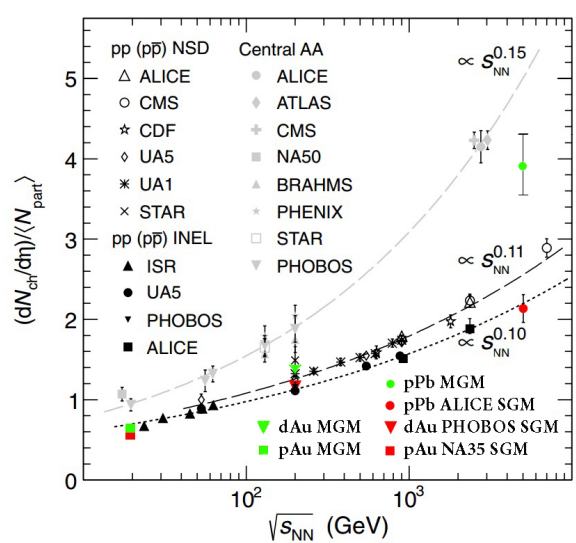

b)

Figure 3: a) The average number of participants, $N_{\text {part }}$, vs. impact parameter for p-Pb collisions at $\sqrt{s}=5.04$ TeV obtained by using SGM (blue points) and MGM with $k=0.35$ (red points). b)The pseudorapidity density of charge particles in proton-nucleus collisions normalized to the average number of participants, $N_{\text {part }}$, for different colliding systems and energies.( All data compilation is from [6] except the corrected green points obtained in the present paper). Red points - normalized data in p- $\mathrm{Pb}, \mathrm{p}$-Au and d-Au collisions using SGM [6]. Green points - recalculated values obtained in the present study from MGM with $k=0.35$.

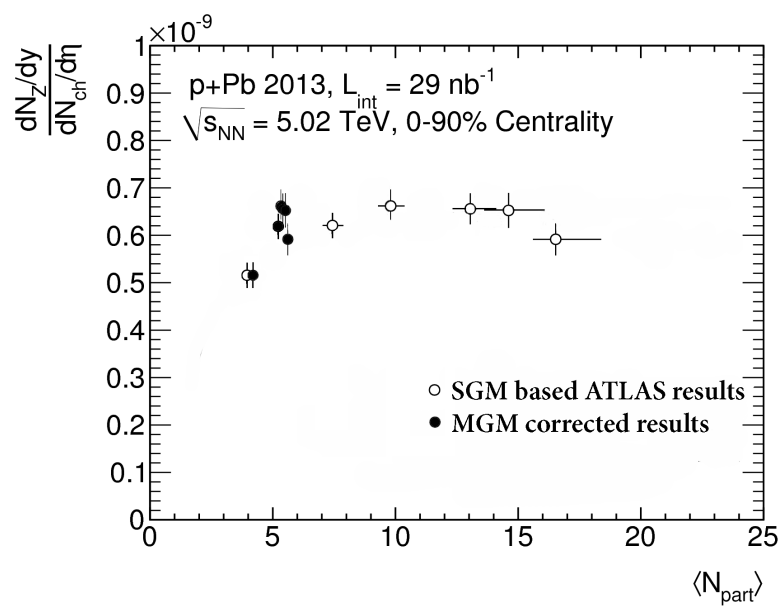

Figure 4: The ratio of Z-bosons yield to full multiplicity versus $N_{\text {part }}$ Open circles - ATLAS data where standard Glauber model estimates were used[11]. Black circles - recalculated data by MGM with $k=0.35$.

However, the application of MGM brings considerable reduction in the number of $N_{\text {part }}$ (see black circles in the Figure 4) thus breaking conclusions on scaling done in [11].

\section{Conclusions}

Modified Glauber Model (MGM) with a single parameter $k$, fixed in the analysis, describes the multiplicity data for all centrality intervals in a broad range of $\mathrm{Au}-\mathrm{Au}$ and $\mathrm{Pb}-\mathrm{Pb}$ collision energies (from $\sqrt{s}=19 \mathrm{GeV}$ to $\sqrt{s}=2.76 \mathrm{TeV}$ ). The MGM predicts considerable nucleon stopping effects in 
the soft part of $p_{\mathrm{T}}$-spectra that should be taken into account in pA collisions at the LHC energy. Similar significant change of understanding the experimental data could be referred to the ratio of Z-bosons yields and charged-particle multiplicity breaking the conclusions on scaling with the number of participants in $\mathrm{p}-\mathrm{Pb}$ collisions at the LHC.

\section{References}

[1] Dmitri Kharzeev and Marzia Nardi, Physics Letters B. 05/2001. arXiv: 001202025,2001

[2] M. Miller, Kl. Reygers, St. Sanders, P. Steinberg, Glauber Modeling in High Energy Nuclear Collisions, arXiv:nucl-ex/0701025v1, 2007.

[3] F. Abe et al. (The CDF Collaboration), Phys.Rev. D41 (1990) 2330.

[4] G. Feofilov, A. Ivanov, Journal of Physics G CS, 5, (2005) 230-237.

[5] T. Drozhzhova, G. Feofilov, V. Kovalenko, A. Seryakov, PoS(QFTHEP 2013)053, Geometric properties and charged particles yields behind Glauber model in high energy pA and nucleu-nucleus collisions, 2013.

[6] ALICE Collaboration, CERN-PH-EP-2012-307, 2012, arXiv:1210.3615v2.

[7] TOTEM Collaboration,EPL, 96, 2011, 21002.

[8] PHOBOS Collaboration, PRL, 2003, arXiv:nucl-ex/0301017.

[9] ALICE Collaboration, Phys. Lett. B 726, 2013, 610-622, arXiv:1304.0347v2.

[10] ALICE Collaboration, Phys. Rev. Lett. 105, 2010, 252301.

[11] ATLAS Collaboration, ATLAS-CONF-2014-020, 2014.

[12] J.F. Grosse-Oetringhaus, K. Reygers, arXiv:0912.0023v2 [hep-ex], 2010. 